\title{
FILTRACIÓN MARGINAL CON IONÓMERO DE VIDRIO Y COMPÓMERO EN MOLARES TEMPORARIOS EN PACIENTES DE 5-10 AÑOS
}

\section{A comparative evaluation of marginal leakage between glass ionomer and compomer in patients aged 5-10 years}

\author{
Gladys Eugenia Moreno Morejón ${ }^{1}$, Paola Nataly Campoverde Córdova ${ }^{2}$, Juan Carlos Cabrera Ortiz ${ }^{3}$, Viviana \\ Carolina Calle Cabrera ${ }^{4}$ Cinthya Coralia Salamea Guevara ${ }^{4}$. \\ ${ }^{1}$ Docente de la Universidad de Cuenca. Facultad de Odontología. Cuenca-Ecuador. \\ 2 Odontóloga general. Chordeleg- Ecuador. \\ 3 Odontólogo general del Seguro Social Campesino. Pasaje- Ecuador. \\ ${ }^{4}$ Estudiante de la Universidad de Cuenca. Facultad de Odontología. Cuenca- Ecuador. \\ *gladys.moreno@ucuenca.edu.ec
}

\section{Resumen}

Objetivos: El objetivo de esta investigación fue comparar las características clínicas entre el ionómero de vidrio (Ionolux, Vocco) y el compómero (TwinkyStar, Vocco) en molares temporales para evitar el filtrado marginal en niños de 5-10 años. Materiales y métodos: Estudio de cohorte, prospectivo, con población fija y de enfoque cuantitativo. La muestra estuvo constituida por 22 pacientes de 5 a 10 años de la Clínica de Odontopediatría de la Facultad de Odontología de la Universidad de Cuenca. Se realizaron 44 obturaciones dos en cada participante, una a base de ionómero vítreo fotopolimerizable y otra a base de compómero, con múltiples controles durante 5 meses. Las evaluaciones clínicas siguieron los criterios modificados de la United State Public Health Service (USPHS). Los datos fueron procesados en EXCEL y SPSS con la prueba Tau C de Kendall. Resultados: Las características clínicas entre el ionómero vítreo y el compómero fueron similares, no se observaron diferencias estadísticamente significativas en relación a integridad marginal, decoloración, forma anatómica, presencia de caries marginal, textura superficial y sensibilidad postoperatoria entre ellos. El ionómero vítreo mostró diferencias estadísticamente significativas en cuanto a integridad marginal (p 0,029) entre los tres a los cinco meses. Conclusiones: Ninguno de estos materiales produce sensibilidad posoperatoria. Los dos materiales presentan características aceptables de integridad marginal y textura superficial por lo cual su uso en dientes deciduos es recomendado.

Palabras clave: Compómeros, cementos de ionómero vítreo, rehabilitación bucal, odontopediatría.

\begin{abstract}
Aim: The aim of this research was to compare the clinical features between glass ionomer (Ionolux, Vocco) versus compomer (TwinkyStar, Vocco) in temporary molars to avoid marginal leakage in children aged 5-10 years. Results: A cohort study, prospective, with settled population and quantitative approach. The sample consisted of 22 patients aged 5 to 10 years from Cuenca University School of Dentistry. 44 seals were made two in each participant, one based on glass ionomer and another based on compomer with multiple controls for 5 months. Clinical evaluations followed the modified criteria of the United State Public Health Service (USPHS). Data were processed in EXCEL and SPSS with the Kendall Tau C test. Conclusions: Both materials have acceptable characteristics of marginal integrity and surface texture, therefore, their use in deciduous teeth is recommended. None of these materials produced postoperative sensitivity.
\end{abstract}

Key words: Compomers, glass ionomer cements, oral rehabilitation, pediatric dentistry.

\section{Introducción}

Los materiales de restauración dental son de gran interés profesional debido a sus propiedades físicas, químicas y mecánicas, en procura de encontrar la similitud con la estructura dental, lograr mayor adaptación, capacidad de retención, resistencia, alcanzar una mayor longevidad o 
conseguir la estética deseable por los pacientes. El éxito de los materiales de restauración; es decir la integridad marginal o sellado hermético, evitará el ingreso de fluidos y bacterias de la boca, previniendo entre otras cosas, la caries dental, el reemplazo de las restauraciones y la subsecuente pérdida de las estructuras dentarias. ${ }^{1,2}$

La infancia es una etapa crítica para la salud bucal, debido principalmente a la presencia de caries dental por factores dietéticos o ambientales o a los defectos de esmalte 3. De acuerdo a una investigación a nivel de Latinoamérica y del Caribe realizada por Magaña et al, la caries afecta a un $80 \%$ de la población infantil en familias de bajo nivel socioeconómico causando un foco de infección en la boca y la disminución de la calidad de vida. ${ }^{4}$

La Organización Mundial de la Salud (OMS), ${ }^{5}$ recomienda para la atención de la salud bucal en niños, la restauración dental atraumática, una técnica en gran parte libre de dolor, la cual consiste en eliminar manualmente el tejido carioso y restaurar la pieza afectada evitando el uso de anestesia local, lo cual es efectivo realizarla con ionómero de vidrio o con algún tipo de resina. ${ }^{4,6}$

Los cementos de ionómero de vidrio han presentado características positivas como en su adhesión, manipulación, aplicación, liberación lenta de flúor y su efecto anticariogénico, las cuales han propiciado su uso durante mucho tiempo con resultados satisfactorios; tal es el caso observado por Fereira et $\mathrm{al}^{6}$ en niños de 3 a 5 años con una tasa de supervivencia de $88,97 \%$, disminución de la incidencia de lesiones cariosas y menor necesidad de retratamientos o, como reporta Zilberman ${ }^{7}$ en molares decíduos por la continua transferencia de iones entre el ionómero de vidrio, el esmalte y la dentina optimizando la función bactericida y la longevidad de la restauración en conjunto con el calcio. Por otro lado, los compómeros, son resinas reforzadas fotopolimerizables; poseen características similares a los ionómeros con la ventaja de facilidad de colocación y mayor liberación de fluoruro en el proceso inicial. 8,10

Durante los últimos años se han realizado investigaciones in vitro o estudios de cohorte a fin de comparar la eficacia de un elemento frente a otro; se ha observado, por ejemplo, en Olegario da Costa ${ }^{11}$ al comparar entre el carbómero de vidrio (CAR - Glass Carb omer - GCP Dental), el compómero (COM - Dyract Extra - Dentsply), y el cemento de ionómero de vidrio (GIC - Fuji IX Extra - GC Corp), en una muestra de 286 niños de 5 a 7 años, por medio del análisis de supervivencia de Kaplan-Meier y la prueba de Log-Rank, que la tasa de supervivencia de las restauraciones con carbómero fue del $3,3 \%$, por debajo del compómero $61,3 \%$ e ionómero $62,6 \%$; en Webman et al. ${ }^{12}$ se observó que en una muestra de 427 obturaciones en molares primarios, en un período de 3 años, hubo una tasa de supervivencia del ionómero de vidrio de 97,42\% frente a una tasa de fracaso anual del $0,86 \%$, una situación favorable con las tasas de éxito publicadas previamente de otros materiales restauradores estándar como amalgamas o compómeros; en Borges et al, ${ }^{13}$ en una muestra constituida por 43 niños de 7 a 10 años, por medio del análisis de supervivencia de Kaplan-Meier y la prueba de Log-Rank, al comparar entre el cemento a base de ionómero de vidrio económico (Vitromolar - Nova DFL) y una resina compuesta de nanopartículas (Filtek Z350 XT - 3M ESPE), se observó que las tasas de sobrevivencia de la resina compuesta $(93,7 \%)$ fue mucho más alta que la del ionómero de vidrio de bajo costo $(62,2 \%)$, además, el análisis estadístico reveló diferencias significativas entre los materiales, mostrando un mejor comportamiento de la resina $(H R=0,15$; IC 0,03 a $0,68 ; \mathrm{p}=0,014)$.

Esta investigación tiene por objetivo comparar las características clínicas entre el ionómero vítreo (Ionolux, Vocco) y el compómero (TwinkyStar, Vocco) en molares temporales para evitar el filtrado marginal en niños de 5-10 años.

\section{Materiales y métodos}

Se realizó un estudio comparativo, prospectivo, con población fija y de enfoque cuantitativo. El universo incluyó a niños y niñas de 5 a 10 años que asistieron a la Clínica de Odontopediatría de la Facultad de Odontología de la Universidad de Cuenca durante cinco meses en el período 2014-2015. La muestra fue propositiva; es decir, por conveniencia; estuvo constituida por 44 molares de 22 pacientes (dos molares de cada uno). Respecto a la selección de la muestra, se consideró la disponibilidad de los pacientes, la facilidad de seguimiento y otras ventajas logísticas tal como se recomienda y se ha practicado en el caso de estudios de cohorte en otras investigaciones. ${ }^{14,15}$

Los criterios de inclusión fueron: pacientes entre los 5 a 10 años que presenten diagnóstico de caries primaria o secundaria en molares temporarios que requieran una preparación cavitaria clase I de Black y cuyos padres o tutores legales firmen el asentimiento informado. Los criterios de exclusión fueron: pacientes entre los 5 a 10 años que no presenten caries primaria o secundaria en molares temporarios, o que presentando, los representantes no autorizaron su participación en la investigación.

Para el control y evaluación se usaron los criterios modificados del Servicio de Salud pública de los Estados Unidos (USPHS, siglas en inglés) debido a la validez en la determinación de la supervivencia entre obturaciones en distintos periodos de tiempo. ${ }^{16-18}$

Los molares fueron debidamente catalogados mediante el Sistema Internacional para la Detección y Evaluación de 


\begin{tabular}{|c|c|c|c|}
\hline Categoría & \multicolumn{2}{|c|}{$\begin{array}{l}\text { Escala de calificación } \\
\text { Aceptable / Inaceptable }\end{array}$} & Criterio \\
\hline \multirow{4}{*}{ Integridad Marginal } & Alpha (A) & & $\begin{array}{l}\text { Ausencia de brecha visible y retención en la } \\
\text { superficie mediante examen con explorador. }\end{array}$ \\
\hline & Bravo (B) & & $\begin{array}{l}\text { Presencia de brecha visible y retención sobre la } \\
\text { superficie mediante examen con explorador. }\end{array}$ \\
\hline & & & $\begin{array}{l}\text { Dentina expuesta con ingreso del explorador en } \\
\text { la brecha visible. }\end{array}$ \\
\hline & & Delta (D) & $\begin{array}{l}\text { Movilidad, fractura total o parcial, pércida de la } \\
\text { restauración. }\end{array}$ \\
\hline \multirow{3}{*}{$\begin{array}{l}\text { Decoloración } \\
\text { Marginal }\end{array}$} & Alpha (A) & & Sin cambio de color. \\
\hline & Bravo (B) & & Cambio de color y no ingresa en el margen. \\
\hline & & Charlie (C) & Cambio de color marginal. \\
\hline \multirow{3}{*}{ Forma Anatómica } & Alpha (A) & & $\begin{array}{l}\text { Continuidad y forma anatómica existente de la } \\
\text { restauración. }\end{array}$ \\
\hline & Bravo (B) & & $\begin{array}{l}\text { Discontinuidad, forma anatómica existente de la } \\
\text { restauración y } \sin \text { dentina expuesta. }\end{array}$ \\
\hline & & Charlie (C) & Pérdida de material que expone la dentina. \\
\hline \multirow{2}{*}{ Caries secundaria } & Alpha (A) & & Ausencia de caries entre restauración- diente. \\
\hline & & Charlie (C) & Presencia de caries entre restauración- diente. \\
\hline \multirow{3}{*}{ Textura supericial } & Alpha (A) & & Superície equivalente al del esmalte pulido. \\
\hline & Bravo (B) & & Supericie con mayor rugosidad que esmalte. \\
\hline & & Charlie (C) & Superiicie rugosa (áspera). \\
\hline \multirow{3}{*}{$\begin{array}{l}\text { Sensibilidad } \\
\text { postoperatoria }\end{array}$} & Alpha (A) & & Ausente. \\
\hline & & Bravo (B) & Sensibilidad que atenúa. \\
\hline & & Charlie (C) & Sensibilidad constante. \\
\hline
\end{tabular}

Caries (ICDAS, siglas en inglés). Participaron dos operadores, (estudiantes de décimo ciclo de la Facultad de Odontología de la Universidad de Cuenca). La observación clínica, la certificación de anatomía y brecha marginal de las obturaciones se llevó a cabo por profesionales de alto prestigio en la zona de estudio, los doctores Pablo Tamariz y Juan Ordoñez, quienes fueron previamente calibrados de acuerdo a los criterios modificados de la USPHS. Se realizaron 44 obturaciones, dos en cada participante, una con ionómero de vidrio (Ionolux, Vocco) y otra con compómero (TwinkyStar, Vocco). La profundidad de la cavidad se determinó por la extensión de la lesión cariosa, con mínima invasión. Se realizó el aislamiento relativo del maxilar superior e inferior, el bloqueo de la salida del conducto de Stenon y la extracción de saliva. La técnica de restauración fue guiada por las instrucciones de uso brindadas por el fabricante en el caso de ambos materiales. Los controles se realizaron de manera inicial, a los tres y a los cinco meses.

\section{Resultados}

La edad promedio de los pacientes tratados fue de 7,27 años. Se realizaron 44 obturaciones: 22 con compómero y 22 con ionómero de vidrio.

El control inicial reportó que la totalidad de obturaciones se encontraban dentro del código Alpha (A) de los criterios USPHS; es decir se observó integridad marginal, no hubo retención del explorador en el margen de la restauración, la restauración fue continua con forma anatómica existente, no hubo cambio de color, no se presentó caries marginal y la textura superficial fue equivalente a la del esmalte pulido.

El control a los tres meses no registró variación de los criterios en el compómero; en el caso del ionómero se presentaron cambios en la decoloración marginal código B $(9,1 \%)$, forma anatómica código B $(13,6 \%)$ y textura superficial código B $(4,5 \%)$; no se registraron diferencias estadísticamente significativas en ninguno de los criterios (Tabla2).

El control a los cinco meses reportó variación con el compómero en caries secundaria código C (4,5\%), en el ionómero se presentaron cambios en la decoloración marginal B $(4,5 \%)$, forma anatómica código B $(9,1 \%)$ y código C $(4,5 \%)$ y textura superficial código B $(4,5 \%)$ (Tabla 2$)$.

Se observaron diferencias estadísticamente significativas respecto al criterio de integridad marginal con el ionómero de vidrio $(\mathrm{p}=0,029)$ (Tabla 2$)$. No se presentó sensibilidad posoperatoria en ninguno de los controles para los dos materiales de obturación. De acuerdo a los criterios USPHS no se presentaron diferencias estadísticamente significativas entre el ionómero vítreo y el compómero a los tres meses ni a los cinco meses (Tabla 3).

\section{Discusión}

La presente investigación observó diferencias mínimas entre los dos materiales (ionómero vítreo y compómero), no hubo diferencias estadísticamente significativas en la mayoría de los resultados.

El ionómero de vidrio a los cinco meses presentó una diferencia estadísticamente significativa en cuanto al criterio de integridad marginal, lo que determina efectivamente el éxito del material, ya que el sellado hermético evitará el ingreso de fluidos y bacterias de la boca, la formación de caries dental, el subsecuente reemplazo de las restauraciones y la pérdida de las estructuras dentarias, tal como lo observaron Acurio et $\mathrm{al}^{1}$ y Herrera et al. $^{2}$ Sin embargo, es importante señalar la importancia de lo observado por Zilberman ${ }^{7}$ y Makkai et al, ${ }^{10}$ es decir, considerar la importancia de la mineralización posterior a la restauración como la aplicación de calcio y el uso de un dentífrico fluorado para asegurar la longevidad de la restauración.

En cuanto a caries secundaria clínicamente diagnosticadas, no se observaron diferencias significativas presentándose el 95,5\% en el criterio A y solo el $4,5 \%$ en el criterio B para el compómero; de esta manera, la posibilidad de intervenir tempranamente y de forma preventiva con los materiales utilizados en el presente estudio, contribuiría a la disminución del foco de infección en la boca y al aumento de la calidad de vida, sobre todo en población infantil de bajo nivel socioeconómico. ${ }^{4,5}$

Al comparar la efectividad entre ambos materiales en la presente investigación, el compómero presentó ligeramente mejores condiciones que el ionómero de vidrio, lo que se considera un comportamiento atribuido a la calidad del material, sobre todo por evidenciar altos valores iniciales de liberación de fluoruro; ${ }^{10}$ sin embargo, esta observación contradice, sin considerar las marcas, el costo de los materiales y las diferencias técnicas de evaluación utilizadas en otras investigaciones, a lo reportado en otros estudios, como en 
Tabla 2: Frecuencia, porcentaje y prueba de significancia del compómero y ionómero frente a los criterios USPHS a los 3 y 5 meses.

\begin{tabular}{|c|c|c|c|c|c|c|c|c|c|c|c|}
\hline \multirow{3}{*}{ Criterios USPHS } & \multirow[b]{3}{*}{ 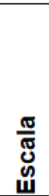 } & \multicolumn{5}{|c|}{ Compómero } & \multicolumn{5}{|c|}{ Ionómero } \\
\hline & & \multicolumn{2}{|c|}{3 Meses } & \multicolumn{2}{|c|}{5 Meses } & \multirow[t]{2}{*}{$\mathbf{p}$} & \multicolumn{2}{|c|}{3 Meses } & \multicolumn{2}{|c|}{5 Meses } & \multirow[t]{2}{*}{$\mathbf{P}$} \\
\hline & & No. & $\%$ & No. & $\%$ & & No. & $\%$ & No. & $\%$ & \\
\hline \multirow{4}{*}{ Integridad Marginal } & $\bar{A}$ & 20 & 90,9 & 20 & 90,9 & \multirow{4}{*}{0,099} & 19 & 86,4 & 18 & 81,8 & \multirow{4}{*}{0,029} \\
\hline & $B$ & 2 & 9,1 & 1 & 4,5 & & 3 & 13,6 & 2 & 9,1 & \\
\hline & C & 0 & 0 & 1 & 4,5 & & 0 & 0 & 1 & 4,5 & \\
\hline & $D$ & 0 & 0 & 0 & 0 & & 0 & 0 & 1 & 4,5 & \\
\hline \multirow{4}{*}{ Decoloración marginal } & A & 22 & 100 & 22 & 100 & & 20 & 90,9 & 20 & 90,9 & \multirow{4}{*}{0,281} \\
\hline & $B$ & 0 & 0 & 0 & 0 & & 2 & 9,1 & 1 & 4,5 & \\
\hline & C & 0 & 0 & 0 & 0 & - & 0 & 0 & 0 & 0 & \\
\hline & * & 0 & 0 & 0 & 0 & & 0 & 0 & 1 & 4,5 & \\
\hline \multirow{4}{*}{ Forma anatómica } & A & 22 & 100 & 22 & 100 & & 19 & 86,4 & 18 & 81,8 & \multirow{4}{*}{0,103} \\
\hline & $B$ & 0 & 0 & 0 & 0 & & 3 & 13,6 & 2 & 9,1 & \\
\hline & C & 0 & 0 & 0 & 0 & - & 0 & 0 & 1 & 4,5 & \\
\hline & * & 0 & 0 & 0 & 0 & & 0 & 0 & 1 & 4,5 & \\
\hline \multirow[b]{2}{*}{ Caries secundaria } & A & 22 & 100 & 21 & 95,5 & & 22 & 100 & 22 & 100 & \multirow[b]{2}{*}{-} \\
\hline & C & 0 & 0 & 1 & 4,5 & - & 0 & 0 & 0 & 0 & \\
\hline \multirow{5}{*}{ Textura superficial } & $A$ & 22 & 100 & 22 & 100 & & 21 & 95,5 & 20 & 90,9 & \multirow{4}{*}{0,457} \\
\hline & $B$ & 0 & 0 & 0 & 0 & & 1 & 4,5 & 1 & 4,5 & \\
\hline & C & 0 & 0 & 0 & 0 & - & 0 & 0 & 0 & 0 & \\
\hline & * & 0 & 0 & 0 & 0 & & 0 & 0 & 1 & 4,5 & \\
\hline & $A$ & 22 & 100 & 22 & 100 & & 22 & 100 & 22 & 100 & \multirow{3}{*}{-} \\
\hline \multirow{2}{*}{$\begin{array}{l}\text { Sensibilidad } \\
\text { posoperatoria }\end{array}$} & $B$ & 0 & 0 & 0 & 0 & - & 0 & 0 & 0 & 0 & \\
\hline & $C$ & 0 & 0 & 0 & 0 & & 0 & 0 & 0 & 0 & \\
\hline
\end{tabular}

${ }^{*}$ Caso perdido por desalojo de la obturación.

Olegario da Costa $^{11}$ una tasa de supervivencias del ionómero de $62,6 \%$ superior al compómero de $61,3 \%$ o en Webman et $\mathrm{al}^{12}$ en donde el ionómero de vidrio presentó una tasa de éxito de $97,42 \%$ frente a otras tasas normalizadas de materiales como amalgamas o compómeros.

Sin duda la calidad de los materiales reflejados en los costos y las diferencias técnicas para la evaluación van a arrojar diferencias en los resultados, al igual que si se consideran las condiciones sanitarias y de salubridad de la población considerada dentro de la investigación, así también lo manifestaron
Suárez y Torres $^{3}$ y Borges et al. ${ }^{13}$

\section{Conclusiones}

Esta investigación no reportó diferencias estadísticamente significativas entre las obturaciones con ionómero de vidrio y compómero en cuanto a integridad marginal en los molares temporales de niños de 5 a 10 años evaluados clínicamente; por lo tanto, se concluye que estos materiales son aptos para la restauración, ya que sus cualidades incluyen efecto antibacterial y anticariogénico que prolonga su vida 
Tabla 3: Prueba de significancia entre compómero y ionómero a los 3 y 5 meses.

\begin{tabular}{|c|c|c|c|c|c|c|c|c|c|c|c|}
\hline \multirow{3}{*}{ Criterios USPHS } & \multirow[b]{3}{*}{$\begin{array}{l}\frac{\pi}{\pi} \\
\text { யூ }\end{array}$} & \multicolumn{4}{|c|}{3 meses } & \multicolumn{6}{|c|}{5 meses } \\
\hline & & \multicolumn{2}{|c|}{ Compómero } & \multicolumn{2}{|c|}{ lonómero } & \multirow[t]{2}{*}{$\mathrm{p}$} & \multicolumn{2}{|c|}{ Compómero } & \multicolumn{2}{|c|}{ Ionómero } & \multirow[t]{2}{*}{$\mathbf{P}$} \\
\hline & & No. & $\%$ & No. & $\%$ & & No. & $\%$ & No. & $\%$ & \\
\hline \multirow{4}{*}{ Integridad Marginal } & $\bar{A}$ & 20 & 90,9 & 19 & 86,4 & \multirow{4}{*}{0,357} & 20 & 90,9 & 18 & 81,8 & \multirow{4}{*}{0,416} \\
\hline & B & 2 & 9,1 & 3 & 13,6 & & 1 & 4,5 & 2 & 9,1 & \\
\hline & $c$ & 0 & 0 & 0 & 0 & & 1 & 4,5 & 1 & 4,5 & \\
\hline & $D$ & 0 & 0 & 0 & 0 & & 0 & 0 & 1 & 4,5 & \\
\hline \multirow{4}{*}{ Decoloración marginal } & A & 22 & 100 & 20 & 90,9 & \multirow{4}{*}{-} & 22 & 100 & 20 & 90,9 & \\
\hline & B & 0 & 0 & 2 & 9,1 & & 0 & 0 & 1 & 4,5 & \\
\hline & $C$ & 0 & 0 & 0 & 0 & & 0 & 0 & 0 & 0 & \\
\hline & * & 0 & 0 & 0 & 0 & & 0 & 0 & 1 & 4,5 & \\
\hline \multirow{4}{*}{ Forma anatómica } & A & 22 & 100 & 19 & 86,4 & \multirow{4}{*}{-} & 22 & 100 & 18 & 81,8 & \\
\hline & $B$ & 0 & 0 & 3 & 13,6 & & 0 & 0 & 2 & 9,1 & \\
\hline & $c$ & 0 & 0 & 0 & 0 & & 0 & 0 & 1 & 4,5 & \\
\hline & * & 0 & 0 & 0 & 0 & & 0 & 0 & 1 & 4,5 & \\
\hline \multirow[b]{2}{*}{ Caries secundaria } & A & 22 & 100 & 22 & 100 & \multirow[b]{2}{*}{-} & 21 & 95,5 & 22 & 100 & \multirow[b]{2}{*}{-} \\
\hline & $c$ & 0 & 0 & 0 & 0 & & 1 & 4,5 & 0 & 0 & \\
\hline \multirow{5}{*}{ Textura superficial } & A & 22 & 100 & 21 & 95,5 & & 22 & 100 & 20 & 90,9 & \multirow{4}{*}{ - } \\
\hline & B & 0 & 0 & 1 & 4,5 & & 0 & 0 & 1 & 4,5 & \\
\hline & $c$ & 0 & 0 & 0 & 0 & - & 0 & 0 & 0 & 0 & \\
\hline & * & 0 & 0 & 0 & 0 & & 0 & 0 & 1 & 4,5 & \\
\hline & $A$ & 22 & 100 & 22 & 100 & & 22 & 100 & 22 & 100 & \multirow{3}{*}{ - } \\
\hline \multirow{2}{*}{$\begin{array}{l}\text { Sensibilidad } \\
\text { posoperatoria }\end{array}$} & $B$ & 0 & 0 & 0 & 0 & - & 0 & 0 & 0 & 0 & \\
\hline & $C$ & 0 & 0 & 0 & 0 & & 0 & 0 & 0 & 0 & \\
\hline
\end{tabular}

útil garantizando un servicio adecuado de la clínica de odontopediatría de la Facultad de Odontología.

El hecho de que ambos materiales no tuvieron diferencias estadísticamente significativas en los criterios de decoloración marginal, forma anatómica, formación de caries secundaria, textura superficial y sensibilidad postoperatoria, que fueron de fácil aplicación y no se evidenciaron experiencias traumáticas en la aplicación a los pacientes, se pueden considerar aptos para la aplicación en dientes deciduos.

La experiencia exitosa en la presente investigación, tanto como la observada en otras investigaciones insinúa la importancia de trabajar con productos de buena calidad, al igual que seguir las instrucciones de los fabricantes.

Se consideraron como limitaciones de la presente investigación, el tiempo de evaluación de los materiales de obturación y la muestra reducida; se recomienda prolongar el tiempo de evaluación y ampliar la muestra en futuros estudios .

\section{Recomendaciones}

En posteriores estudios para evitar disminución de la muestra se recomienda considerar el periodo de exfoliación fisiológica de las piezas dentales y realizar experimentos con materiales de diferentes marcas y costos. 
Conflicto de intereses: Los autores declaran no tener con- 13 Borges JI, Olegário IC, Alvarez P de L, Prócida D, Parisotto flicto de intereses.

\section{Referencias}

1 Acurio P, Falcón G, Casas L. Comparación de la resistencia compresiva de resinas convencionales vs resinas tipo Bulk fill. Odontología Vital. 2017;(27):69-77.

2 Herrera S, Sánchez F, Reyes G, Vázquez E, Guerrero J. Microfiltración en restauraciones de resinas realizadas con diferentes sistemas adhesivos estudio In Vitro. Revista Odontológica Latinoamericana. 2016;8(2):41-5.

3 Suárez A, Torres G. Nuevos materiales restauradores con liberación de fúor en manejo rehabilitador de odontopediatría. Reporte de caso. Rev Simiykita [Internet]. 2016 [citado 31 de agosto de 2020];2(2).

4 Magaña AG, Calvillo DH, Cárdenas JM. Caries dental en niños con nivel socioeconómico bajo en América. Ciencia Cierta [Internet]. 2017;(49).

5 OMS/OPS. La Salud Oral de los Niños de Bajos ingresos: Procedimientos para el Tratamiento Restaurativo Atraumático (PRAT) [Internet]. Washington, D.C; 2006 [citado 31 de agosto de 2020]. Report No.: 091024.

6 Fereira JL, Medrano LEC, García IT, Álvarez MA, Paredes S, Montoya MEH. Supervivencia de restauraciones atraumáticas en molares primarios. Contexto Odontológico [Internet]. 13 de agosto de 2018 [citado 31 de agosto de 2020];6(11).

7 Zilberman U. Ion exchanges between glass-ionomer restorative material and primary teeth components-an in vivo study. Oral Biology and Dentistry [Internet]. 2014 [citado 31 de agosto de 2020];1(2).

8 Colmenares AK, Peña BS, Molina EA, Agelvis MJ. Comportamiento de los compómeros y composites en restauraciones de dientes posteriores: una revisión sistemática. Revista Venezolana de Investigación Odontológica. 8 de julio de 2016;4(2):234-52.

9 Rosero J, Mendoza F, Rosero J, Hernández V. Grado de microfiltración en restauraciones con ionomeros vidrio de base variando la secuencia en los procedimientos de restauración. Polo del Conocimiento. 2017;2(6):1016-39.

10 Makkai Z, Szekely M, Fazakas Z, Nagy L, Barta K, Mathe B. Fluoride Release and Uptake Capability of Glassionomer Cements and Compomers Used as Dental Restorative Materials I Request PDF. ResearchGate. 2019;56:54853.

11 Olegário da Costa IC. Longevidade de restaurações ART em cavidades ocluso-proximais utilizando três tipos de materiais em molares decíduos. Estudo Clínico Randomizado. 2015;69-69.

12 Webman M, Mulki E, Roldan R, Arevalo O, Roberts JF, Garcia F. A Retrospective Study of the 3-Year Survival Rate of Resin-Modified Glass-Ionomer Cement Class II Restorations in Primary Molars. Journal of Clinical Pediatric Dentistry. 22 de diciembre de 2015;40(1):8-13.
T, Petorossi JC. Sobrevida de resina compuesta y cemento de ionómero de vidrio de bajo costo para TRA: 1 año de seguimiento de un ensayo clínico aleatorizado. RO. 6 de agosto de 2018;20(1):88-106.

14 Bechere M, Vecchi G, Lellis D, Ferraro A. Excess body weight in children may increase the length of hospital stay. Clinics. 2015;70(2):87-90.

15 Szlachcic Y, Adkins RH, Govindarajan S, Cao Y, Krause JS. Cardiometabolic changes and disparities among persons with spinal cord injury: a 17-year cohort study. Top Spinal Cord Inj Rehabil. 2014;20(2):96-104.

16 Santiago SL, Passos VF, Vieira AHM, Navarro M, Lauris J, Franco E. Two-year clinical evaluation of resinous restorative systems in non-carious cervical lesions. Brazilian Dental Journal. 2010;21(3):229-34.

17 Arhun N, Celik C, Yamanel K. Clinical evaluation of resinbased composites in posterior restorations: two-year results. ResearchGate. 2010;35(4):397-404.

18 Jyothi K, Annapurna S, Kumar AS, Venugopal P, Jayashankara C. Clinical evaluation of giomer- and resinmodified glass ionomer cement in class $\mathrm{V}$ noncarious cervical lesions: An in vivo study. J Conserv Dent. octubre de 2011;14(4):409-13.

Recibido: 15 de septiembre de 2020

Aceptado: 01 de diciembre de 2020 